\section{RISK ASSESSMENT FOR EARTHQUAKE SURVIVORS: EVALUATION OF GENERATION DIFFERENCE}

\section{To the Editor:}

I have read with much interest the timely paper by Hashmi et al dealing with effects of earthquakes using questionnaires. ${ }^{1}$ The great East Japan disaster struck on March 11, 2011. This disaster encompassed multiple components, including the earthquake, the consequent tsunami, and the nuclear power plant accident. Approximately 20000 persons died or are still missing.

The authors used the Posttraumatic Stress Disorder Checklist (PCL) Scale $^{2}$ and the Hopkins Symptom Checklist (HSCL) scale ${ }^{3}$ to evaluate posttraumatic stress disorder (PTSD) and anxiety-depression in the survivors. They reported that half of the survivors had symptoms of PTSD, and that more than three-fourths of the survivors still had symptoms of anxiety 6 months after the incident. Their research, however, had a cross-sectional design. A continuous followup of the 361 survivors enrolled would have clarified the change in the prevalence of PTSD, anxiety, and depression with time.

Johannesson et al repeatedly used the Impact of Event ScaleRevised (IES-R) ${ }^{4}$ and the General Health Questionnaire, 12 item version (GHQ-12), ${ }^{5}$ to evaluate PTSD and general mental health. ${ }^{6}$ These authors reported on a comparison of the IES-R scores and GHQ-12 scores that were evaluated at 14 months and 3 years, respectively, after a disaster. While the GHQ-12 score increased in the subjects who were 40 years or younger, the score decreased in the subjects who were older than 40 years; the IES-R score decreased in all age groups. The PTSD score did not change in parallel with the general mental health. As a result, special attention should be paid to the younger generation to provide mental support for victims of natural disasters.
Although Hashmi et al classified the survivors in their study into 2 groups by age, using 45 years as the cutoff point, they found no significant contribution of age class on anxiety, depression, or PTSD in the subjects. Based on these findings, I recommend that Hashmi et al conduct a longitudinal follow-up of the survivors to clarify the long-term health effects of the great disaster.

Tomoyuki Kawada, MD

Author Affiliation: Department of Hygiene and Public Health, Nippon Medical School, Tokyo, Japan.

Correspondence: Tomoyuki Kawada, MD, Department of Hygiene and Public Health, Nippon Medical School, 1-1-5 Sendagi, Bunkyo-Ku, Tokyo 113. 8602, Japan (e-mail: kawada@nms.ac.jp).

\section{REFERENCES}

1. Hashmi S, Petraro P, Rizzo T, et al. Symptoms of anxiety, depression, and posttraumatic stress among survivors of the 2005 Pakistani earthquake. Disaster Med Public Health Prep. 2011;5(4):293-299.

2. Ventureyra VA, Yao SN, Cottraux J, Note I, De Mey-Guillard C. The validation of the Posttraumatic Stress Disorder Checklist Scale in posttraumatic stress disorder and nonclinical subjects. Psychother Psychosom. 2002; 71(1):47-53.

3. Halepota AA, Wasif SA. Hopkins Symptoms Checklist 25(HSCL-25) Urdu translation: an instrument for detecting anxiety and depression in torture and trauma victims. J Pak Med Assoc. 2001;51(7):255-257.

4. Weiss DS, Marmar CR. The impact of event scale-revised. In: Wilson JP, Keane TM, eds. Assessing Psychological Trauma and PTSD. New York, NY: Guilford Press; 1997:399-411.

5. Goldberg DP, Gater R, Sartorius N, et al. The validity of two versions of the GHQ in the WHO study of mental illness in general health care. Psychol Med. 1997;27(1):191-197.

6. Johannesson KB, Lundin T, Fröjd T, Hultman CM, Michel PO. Tsunamiexposed tourist survivors: signs of recovery in a 3-year perspective. J Nerv Ment Dis. 2011;199(3):162-169. 